\title{
Tecnologia delle applicazioni digitali per il diabete: benefici, sfide e raccomandazioni. Consensus Report del Diabetes Technology Working Group della European Association for the Study of Diabetes (EASD) e della American Diabetes Association (ADA)
}

\author{
Raffaella Buzzetti ${ }^{1} \cdot$ Luca D$^{\prime}$ Onofrio ${ }^{1}$
}

Pubblicato online: 23 luglio 2020

(c) The Author(s) 2020

Commento a:

Diabetes digital app technology: benefits, challenges, and recommendations. A Consensus Report by the European Association for the Study of Diabetes (EASD) and the American Diabetes Association (ADA) Diabetes Technology Working Group.

G.A. Fleming, J.R. Petrie, R.M. Bergenstal, R.W. Holl, A.L. Peters, L. Heinemann.

Diabetes Care (2020) 43(1):250-260

Ben nota è la rivoluzione, avvenuta nel campo della tecnologia digitale negli ultimi trent'anni, che ha interessato soprattutto i dispositivi mobili. Si è assistito a un aumento di nuovi strumenti medici che oggi affiancano il personale sanitario nello svolgimento del loro lavoro. Oltre a questi strumenti sono state anche sviluppate applicazioni (App) per smartphone di ausilio al paziente e al personale sanitario, non legate a strumenti medici. Proprio a queste App standalone si rivolge l'attenzione di questo documento redatto da due tra le principali società che si occupano di diabete [1], campo dove la tecnologia digitale ha permesso notevoli miglioramenti nella gestione clinica.

Il documento, oltre a fare chiarezza relativamente agli organi cui compete la regolamentazione in questo campo, soprattutto nell'Unione Europea e negli USA, si concentra sulle maggiori criticità e potenzialità di queste App, redigendo alcune raccomandazioni specifiche per il loro miglioramento.

Gli autori individuano nove punti di azione. Il primo riguarda le carenti evidenze scientifiche relative agli studi di

$凶$ R. Buzzetti

raffaella.buzzetti@uniroma1.it

1 Dipartimento di Medicina Sperimentale, Sapienza Università di Roma, Roma, Italia efficacia sull'utilizzo di queste App. Gli studi pubblicati ne riportano spesso un beneficio nel breve termine, ma i lavori di revisione sono concordi in merito alla carenza di dati e obiettivi standardizzati su ampie popolazioni nel lungo termine. Secondo, viene evidenziata la necessità di fornire al personale sanitario formazione e informazione in merito al possibile uso di queste App mediante personale o procedure dedicate, impegno oneroso per una categoria già altamente impegnata. Terzo, viene sottolineato quanto sia importante valutare l'accuratezza e la validità clinica di queste App. Gli autori invitano a considerare i medesimi standard per le App con le medesime finalità, enfatizzando l'importanza di definire chiaramente l'utenza alla quale la App si riferisce (esempio genitori oppure adolescenti) e l'uso della stessa terminologia. Quarto punto affrontato è la problematica tecnologica, auspicando accortezza nel considerare l'impatto della singola App e dei suoi aggiornamenti sul dispositivo mobile. Quinto, gli autori sottolineano la possibilità che queste App possano dialogare facilmente con altre piattaforme informatiche utilizzate dal personale sanitario per un ottimale utilizzo dei dati. Sesto, gli autori sollevano la problematica delle differenti popolazioni, sia in merito all'età (configurazione età-specifica), sia in merito alle condizioni socioeconomiche, con la raccomandazione di non escludere le persone appartenenti alle fasce meno abbienti. Viene poi esortato il personale sanitario, affinché sfrutti questi mezzi per migliorare l'assistenza fornita al paziente. Anche le società scientifiche vengono sollecitate a stilare regolamenti che possano guidare lo sviluppo di questi strumenti. Infine, come ultimo punto viene sottolineata con fermezza l'importanza della salvaguardia della privacy dei dati degli utenti da parte delle aziende che sviluppano queste App.

In conclusione, gli autori invitano a migliorare lo sviluppo e l'uso delle App dedicate alla cura del paziente diabetico, rivolgendosi a tutte le figure interessante a vario titolo in 
questo processo, personale sanitario, mondo accademico ed enti regolatori.

All'alba degli anni Venti del nuovo secolo, questa position statement non solo fa il punto sull'utilizzo della tecnologia mobile nella cura del diabete ma ci esorta in prima persona ad approfondirne la conoscenza e incrementarne l'utilizzo.

Ringraziamenti Open access funding provided by Università degli Studi di Roma La Sapienza within the CRUI-CARE Agreement.

Nota della casa editrice Springer Nature rimane neutrale in riguardo alle rivendicazioni giurisdizionali nelle mappe pubblicate e nelle affiliazioni istituzionali.

Open Access This article is licensed under a Creative Commons Attribution 4.0 International License, which permits use, sharing, adaptation, distribution and reproduction in any medium or format, as long as you give appropriate credit to the original author(s) and the source, provide a link to the Creative Commons licence, and indicate if changes were made. The images or other third party material in this article are included in the article's Creative Commons licence, unless indicated otherwise in a credit line to the material. If material is not included in the article's Creative Commons licence and your intended use is not permitted by statutory regulation or exceeds the permitted use, you will need to obtain permission directly from the copyright holder. To view a copy of this licence, visit http://creativecommons.org/licenses/by/4.0/.

\section{Bibliografia}

1. Fleming GA, Petrie JR, Bergenstal RM et al (2020) Diabetes digital app technology: benefits, challenges, and recommendations. A consensus report by the European Association for the Study of Diabetes (EASD) and the American Diabetes Association (ADA). Diabetes Technol Work Group Diabetes Care 43(1):250-260 